\title{
Asymptotically Stable Walking of a Simple Underactuated 3D Bipedal Robot
}

\author{
Ching-Long Shih* \\ J.W. Grizzle** \\ and \\ C. Chevallereau*** \\ *EE Department, National Taiwan University of Science and Technology, Taipei, Taiwan 106 \\ **Control Systems Laboratory, EECS Department, University of Michigan, Ann Arbor, Michigan 48109-2122 USA \\ *** CNRS, IRCCyN, Nantes Atlantic University, 1 rue de la Noe, 44321 Nantes, cedex 03, France \\ shihcl@mail.ntust.edu.tw_grizzle@eecs.umich.edu $\quad$ Christine.Chevallereau@irccyn.ec-nantes.fr
}

\begin{abstract}
This paper presents a feedback controller that achieves an asymptotically stable, periodic, and fast walking gait for a 3D bipedal robot consisting of 3 -links and passive (unactuated) point-feet. The robot has $6 \mathrm{DOF}$ in the single support phase and four actuators. In addition to the reduced number of actuators, the interest of studying robots with point feet is that the feedback control solution must exploit the robot's natural dynamics in order to achieve balance while walking. We use an extension of the method of virtual constraints, a very successful method for planar bipeds, in order to simultaneously compute a periodic orbit and an autonomous feedback controller that realizes the orbit, for a 3D (spatial) bipedal walking robot. This method allows the computations for the controller design and the periodic orbit to be carried out on a 2-DOF subsystem of the 6-DOF robot model. The linearization of the Poincare map of the closed-loop system proves that the achieved periodic walking motion, at a speed of approximately one and a half body lengths per second, is exponentially stable.
\end{abstract}

Keywords: 3D bipedal robot, dynamic walking, orbital stability, Poincare map, zero dynamics, event-based control

\section{INTRODUCTION}

The primary objective of this paper is to contribute to the feedback control of 3D bipedal robots that do not rely on large feet and slow movement for achieving stability of a walking gait. We assume here an unactuated point contact at the leg end and, for a simple 3-link robot, seek a time-invariant feedback controller that creates an exponentially stable, periodic walking motion. Our approach is based on an extension of the method of virtual constraints, which was developed in $[1,2,3,4]$ for planar robots, and is extended here to the case of spatial robots. Virtual constraints are holonomic constraints on the robot's configuration that are asymptotically achieved through the action of a feedback controller. Their function is to coordinate the evolution of the various links of the robot throughout a stride---which is another way of saying that they reduce the degrees of freedom. By using virtual constraints to achieve link coordination on a bipedal robot, different gaits can be more easily programmed than if the links were coordinated by hardware constraints.

The work most closely related to ours is [5], where the control of a 3D walker was decomposed into the study of its motion in the sagittal plane and the frontal plane; see also [6] for a related decomposition result on control in the frontal plane. The method of virtual constraints was applied in [5] to regulate the sagittal plane motion of the biped and an inverted pendulum approximation of the dynamics was used to design a controller for the frontal plane. An event-based controller was then introduced to synchronize the frequency of the independently designed sagittal and frontal plane controllers. The overall closed-loop system was shown to be stable through simulation and subsequently through experimentation. In our approach, we do not decompose the model into sagittal and frontal plane motions, and coupling of the sagittal and frontal plane dynamics is introduced into the controller from the very beginning.

Other important work on the control of spatial robots includes [7], where the control of a five-link 3D robot with unactuated point feet has been designed on the basis of linearizing the robot's model along a periodic orbit. So that the controller would be time-invariant, the orbit was parameterized with a configuration variable that is strictly monotonic throughout a normal gait, as in $[1,2,3,4]$, before linearization was applied. In [8] and references therein, the analysis of passive spatial bipeds is presented; the emphasis in their work is on energy efficiency and not on feedback control for a wide range of behaviors. The work in $[9,10]$ seeks energy efficiency and a large basin of attraction, though full actuation is assumed; in particular actuation between the leg and ground is assumed in the sagittal and frontal planes, as opposed to the unacuated assumption made here.

To the best of our knowledge, other work on the control of spatial robots either assumes full actuation and/or does not provide significant analysis of the closed-loop system (e.g., [11]).

\section{MODEL}

The 3D bipedal robot discussed in this work is shown in Fig. 1. It consists of three links: a torso and two legs without knees that are terminated with "point-feet." Each hip consists of a revolute joint with 2 degrees of freedom and each degree of freedom is independently actuated. The stance leg is assumed to act as a passive pivot in the sagittal and frontal planes, with no rotation about the z-axis (i.e., no yaw motion), so the leg end is modeled as a point contact with 2 degrees of freedom and no actuation. In total, the biped in the single support phase has 6 degrees of freedom, and there are 2 degrees of underactuation.

The following assumptions are made in this study:

(1) Each link is represented by a point mass located at its midpoint. 
(2) Walking consists of two alternating motion phases: single support and double support.

(3) The double support phase is instantaneous and involves the impact of the swing leg with the ground.

(4) The walking motion is symmetric in steady state.

(5) Walking takes place on a flat surface.

A set of generalized coordinates $q=\left[q_{1}, q_{2}, q_{3}, q_{4}, q_{5}, q_{6}\right]^{T}$ is shown in Fig. 1. Absolute angles $q_{1}, q_{2}$ are roll and pitch angles of the stance leg, respectively, relative angles $q_{3}, q_{4}$ are the joint angles of the stance leg relative to the torso along the $y$-axis and the $\mathrm{x}$-axis, respectively, and relative angles $q_{5}, q_{6}$ are the joint angles of the swing leg relative to the torso along the $\mathrm{x}$-axis and the $\mathrm{y}$ axis, respectively. The coordinates $q_{1}, q_{2}$ are unactuated (passive contact), while $q_{3}, q_{4}, q_{5}, q_{6}$ are independently actuated (active joints).

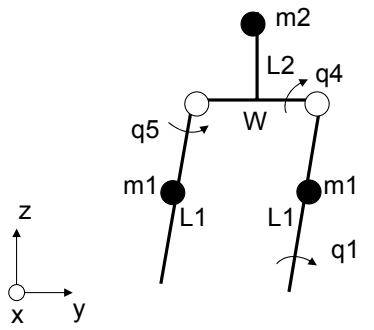

(a) frontal (a.k.a. lateral) plane

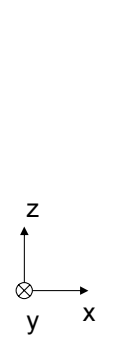

(b) sagittal plane
Figure 1. A three-link 3D point-feet biped. Each link is modeled by a point mass at its center and there is no yaw motion about the stance leg end.

The Euler-Lagrange equations yield the dynamic model for the robot in the single support phase as

$$
D(q)_{6 \times 6} \ddot{q}_{6 \times 1}+C(q, \dot{q})_{6 \times 6} \dot{q}_{6 \times 1}+G(q)_{6 \times 1}=B_{6 \times 4} u_{4 \times 1}=\left[\begin{array}{c}
0_{2 \times 4} \\
I_{4 \times 4}
\end{array}\right] u_{4 \times 1},(1)
$$

where $D(q)$ is the positive-definite mass-inertia matrix, $C(q, \dot{q})$ is the Coriolis matrix, $G(q)$ is the gravity term, $B$ is a constant matrix indicating whether a joint is actuated or not, and $u$ is the vector of input torques. Let $q_{u}=\left[q_{1}, q_{2}\right]^{T}$ denote the unactuated joints and $q_{a}=\left[q_{3}, q_{4}, q_{5}, q_{6}\right]^{T}$ denote the actuated joints. Then Eq. (1) can be rewritten as

$$
\left[\begin{array}{ll}
D_{11} & D_{12} \\
D_{21} & D_{22}
\end{array}\right]\left[\begin{array}{l}
\ddot{q}_{u} \\
\ddot{q}_{a}
\end{array}\right]+\left[\begin{array}{ll}
C_{11} & C_{12} \\
C_{21} & C_{22}
\end{array}\right]\left[\begin{array}{l}
\dot{q}_{u} \\
\dot{q}_{a}
\end{array}\right]+\left[\begin{array}{l}
G_{1} \\
G_{2}
\end{array}\right]=\left[\begin{array}{l}
0_{2 \times 1} \\
u_{4 \times 1}
\end{array}\right] .
$$

Following standard practice in the literature, the double support phase is assumed to be instantaneous. However, it actually consists of two distinct subphases: the impact, during which a rigid impact takes place between the swing foot and the ground, and coordinate relabeling, which is responsible for switching the roles of the two legs after the impact so that same dynamic model can be applied in the single support phase [1]. During the impact, the biped's configuration variables do not change, but the generalized velocities undergo a step change. The derivation of the impact model in double support phase requires 4 additional spatial variables, selected here as the Cartesian coordinates of the stance foot and its orientation, to form an extended set of generalized coordinates $q_{e}=\left[\begin{array}{lllll}q^{T} x_{1} & y_{1} & z_{1} & q_{0, s t}\end{array}\right]^{T}$. Conservation of momentum during the impact process and the swing leg neither slipping nor rebounding at impact yield

$$
\left[\begin{array}{c}
\dot{q}_{e}^{+} \\
F_{2}
\end{array}\right]=\left[\begin{array}{cc}
D_{e} & -E_{2}^{T} \\
E_{2} & 0_{3 \times 3}
\end{array}\right]^{-1}\left[\begin{array}{c}
D_{e} \dot{q}_{e}^{-} \\
0_{3 \times 1}
\end{array}\right],
$$

where $\dot{q}_{e}^{-}$and $\dot{q}_{e}^{+}$are the extended velocities before and after the impact, respectively, $F_{2}$ is the reaction force at the contact point, $D_{e}$ is the extend mass-inertia matrix, and $E_{2}=\frac{\partial}{\partial q_{e}}\left[\begin{array}{llll}x_{2} & y_{2} & z_{2} & q_{0, s w}\end{array}\right]^{T}$ is the Jacobian matrix for the position of the swing foot and its orientation in the plane $x-y$. Analogously to [1], the overall impact model is written as

$$
q^{+}=\Delta_{q}\left(q^{-}\right)
$$

and

$$
\dot{q}^{+}=\Delta_{\dot{q}}\left(\dot{q}^{-}\right)
$$

and is obtained from solving (3) and projecting down to the original configuration variables.

Define state variables as $x=\left[\begin{array}{l}q \\ \dot{q}\end{array}\right]$, and let $x^{+}=\left[\begin{array}{l}q^{+} \\ \dot{q}^{+}\end{array}\right]$and $x^{-}=\left[\begin{array}{c}q^{-} \\ \dot{q}^{-}\end{array}\right]$. Then a complete walking motion of the robot can be expressed as a nonlinear system with impulse effects, as shown in Fig. 2 and written as

$$
\Sigma:\left\{\begin{array}{ll}
\dot{x}=f(x)+g(x) u & x^{-} \notin S \\
x^{+}=\Delta\left(x^{-}\right) & x^{-} \in S
\end{array},\right.
$$

where $S=\left\{(q, \dot{q}) \mid z_{2}(q)=0, x_{2}(q)>0\right\} \quad$ is the switching surface,

$$
f(x)=\left[\begin{array}{c}
\dot{q} \\
D^{-1}(q)(-C(q, \dot{q}) \dot{q}-G(q))
\end{array}\right], g(x)=\left[\begin{array}{c}
0 \\
D^{-1}(q) B
\end{array}\right]
$$

and

$$
x^{+}=\Delta\left(x^{-}\right)=\left[\begin{array}{c}
\Delta_{q}\left(q^{-}\right) \\
\Delta_{\dot{q}}\left(\dot{q}^{-}\right)
\end{array}\right] \text {. }
$$

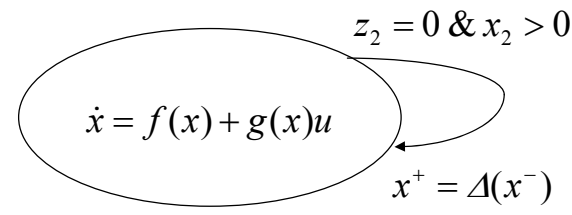

Figure 2. Biped robot dynamic model as a simple hybrid system.

\section{Simultaneous Gait AND Within-Stride CONTROLler DESIGN}

The method of virtual constraints, which has proven very successful in designing feedback controllers for stable walking 
in planar bipeds $[1,2,3,4]$, will be applied to the 3D biped of the previous section. In this method, one holonomic constraint per actuator is proposed in the form of an output that when zeroed by a feedback controller enforces the constraint. The most direct form of the constraint is

$$
y_{4 \times 1}=h(q)=q_{a}-h_{d}(\theta),
$$

where $q_{a}=\left[q_{3}, q_{4}, q_{5}, q_{6}\right]^{T}$ is the vector of actuated coordinates, $\theta=\theta(q)$ is a quantity that is strictly monotonic (i.e., strictly increasing or decreasing) along a typical walking gait, and $h_{d}(\theta)$ is the desired (asymptotic) evolution of the actuated variables as a function of $\theta$. Roughly speaking, $\theta$ is used to replace time in parameterizing a periodic motion of the biped. In a forward walking motion, the speed of the biped is roughly proportional to the rate of change of $q_{2}$, and therefore the choice $\theta=-q_{2}$ is made (the minus sign is used to make $\theta$ strictly increasing over a step).

The next objective is to determine choices for $h_{d}(\theta)$ that are compatible with a periodic walking motion. This task is simplified by noting that enforcing the virtual constraints, $0=y_{4 \times 1}=h(q)$, results in $q_{a}=h_{d}(\theta)$ and reduces the dimension of the dynamics. Indeed, if the output $y$ and its first and second derivatives are all zero (i.e. $y=0, \dot{y}=0$, and $\ddot{y}=0$ ), then the required torque $u^{*}$ can be computed to be

$u^{*}=\left(\frac{\partial h(q)}{\partial q} D^{-1} B\right)^{-1}\left(\frac{\partial^{2} h_{d}(\theta)}{\partial \theta^{2}} \dot{\theta}^{2}(t)+\frac{\partial h(q)}{\partial q} D^{-1}(C(q, \dot{q}) \dot{q}+G)\right)$.

Substituting this into Eq. (2), the dynamic model of the single support phase is now reduced to a low-dimensional autonomous system,

$\left(D_{11}+D_{12} \frac{\partial q_{a}}{\partial q_{u}}\right) \ddot{q}_{u}+\left(C_{11}+C_{12} \frac{\partial q_{a}}{\partial q_{u}}+D_{12} \frac{\partial}{\partial q_{u}}\left(\frac{\partial q_{a}}{\partial q_{u}} \dot{q}_{u}\right)\right) \dot{q}_{u}+G_{1}=0$

which is also called the swing phase zero dynamics. One can clearly see that the dynamic properties of the swing phase zero dynamics depend on the choice of the virtual constraint $0=y=q_{a}-h_{d}(\theta)$, but not on how the constraint is zeroed.

How to determine a choice for $h_{d}(\theta)$ that results in a periodic walking motion is summarized in the next section. This section is concluded by noting that once the virtual constraints are determined, a feedback controller for the robot is suggested by the input-output linearizing controller [12]

$$
u=u^{*}-\left(\frac{\partial h}{\partial q} D^{-1} B\right)^{-1}\left(\frac{K_{p}}{\varepsilon^{2}} y+\frac{K_{d}}{\varepsilon} \dot{y}\right),
$$

which results in $\ddot{y}+\frac{K_{d}}{\varepsilon} \dot{y}+\frac{K_{p}}{\varepsilon^{2}} y=0$. In other words, determining the constraints is equivalent to the design of a feedback controller in the single support phase.

\section{COnstraint Design For a Periodic Gait}

This section is based on [4]. The virtual constraints $q_{a}=h_{d}(\theta)$ are parameterized with Bezier polynomials of order $M$. Let

$$
\begin{aligned}
& h_{d}(\theta)=\left[\begin{array}{llll}
b_{1}(s) & b_{2}(s) & b_{3}(s) & b_{4}(s)
\end{array}\right]^{T} \text {, where } \\
& b_{i}(s)=\sum_{k=0}^{M} \alpha(i, k) \frac{M !}{k !(M-k) !} s^{k}(1-s)^{M-k}, 1 \leq i \leq 4,
\end{aligned}
$$

$s=\frac{\theta-\theta^{+}}{\theta^{-}-\theta^{+}}$is the normalized independent variable, and $\theta^{-}$ and $\theta^{+}$are the values of $\theta$ just before and just after the impact, respectively. Like B-spline curves, a Bezier polynomial is a smooth curve with a local control property. In addition, its position, derivative, and second derivative at the initial and final points are easy to compute as listed below:

(1) Position at the initial and final points

$$
\begin{aligned}
& b_{i}(0)=\alpha(i, 0) \\
& b_{i}(1)=\alpha(i, M)
\end{aligned}
$$

(2) First derivative at the initial and final points

$$
\begin{aligned}
& \frac{\partial b_{i}}{\partial s}(0)=M(\alpha(i, 1)-\alpha(i, 0)) \\
& \frac{\partial b_{i}}{\partial s}(1)=M(\alpha(i, M)-\alpha(i, M-1)) .
\end{aligned}
$$

(3) Second derivative at the initial and final points

$$
\frac{\partial^{2} b_{i}}{\partial s^{2}}(0)=M(M-1)(\alpha(i, 2)-2 \alpha(i, 1)+\alpha(i, 0))
$$

$$
\frac{\partial^{2} b_{i}}{\partial s^{2}}(1)=M(M-1)(\alpha(i, M)-2 \alpha(i, M-1)+\alpha(i, M-2))
$$

The above properties are quite useful when considering the effect of an impact arising in the double-support phase. Indeed, let $\left(q^{-}, \dot{q}^{-}\right)$and $\left(q^{+}, \dot{q}^{+}\right)$be the joint angles and velocities of the biped before and after the impact, respectively. Then according to the impact model, in order to have a periodic solution, the coefficients of the Bezier polynomials need to satisfy the following equations:

$$
\begin{aligned}
& \alpha_{0}=h_{d}\left(\theta^{+}\right)=q_{a}^{+} \\
& \alpha_{1}=q_{a}^{+}+\frac{\theta^{-}-\theta^{+}}{M} \frac{\partial h_{d}}{\partial \theta}\left(\theta^{+}\right)=q_{a}^{+}+\frac{\theta^{-}-\theta^{+}}{M} \frac{\dot{q}_{a}^{+}}{\dot{\theta}^{+}} \\
& \alpha_{M-1}=q_{a}^{-}-\frac{\theta^{-}-\theta^{+}}{M} \frac{\partial h_{d}}{\partial \theta}\left(\theta^{-}\right)=q_{a}^{-}-\frac{\theta^{-}-\theta^{+}}{M} \frac{\dot{q}_{a}^{-}}{\dot{\theta}^{-}} \\
& \alpha_{M}=h_{d}\left(\theta^{-}\right)=q_{a}^{-},
\end{aligned}
$$

where $\alpha_{k}=\left[\begin{array}{llll}\alpha(1, k) & \alpha(2, k) & \alpha(3, k) & \alpha(4, k)\end{array}\right]^{T}, 0 \leq k \leq M$. Therefore, the order of the Bezier polynomials $q_{a}=h_{d}(\theta)$ must be equal to or greater than $3(M \geq 3)$, and the parameters $\alpha_{2}, \cdots, \alpha_{M-2}$ and the initial conditions $\left(q^{-}, \dot{q}^{-}\right) \in S$ are variables that can be freely chosen in the design of a periodic walking motion.

Following [4], the search for a periodic walking motion is cast as a constrained nonlinear optimization problem: Find an initial condition $\left(q^{-}, \dot{q}^{-}\right) \in S$ and virtual constraint 
parameters $\alpha_{2}, \cdots, \alpha_{M-2}$ that minimize the energy consumed per step length, $J=\frac{1}{L} \int_{0}^{T} \frac{1}{2} u^{* T} u^{*} d t$, where $T$ is the walking period and $L$ is the step length, and subject to the following constraints:

(i) $\theta$ is strictly increasing (i.e, $\dot{\theta}>0$ along the solution);

(ii) the swing foot is positioned above the ground $\left(z_{2} \geq 0\right)$;

(iii) a step size constraint;

(iv) a friction constraint, $\sqrt{F_{2 x}^{2}+F_{2 y}^{2}} \leq \mu F_{2 z}$;

(v) the solution is periodic and symmetric with respect to the two legs.

The above procedure can be carried out in MATLAB with the optimization toolbox's FMINCON function as follows:

(1) For the initial state $X_{k}^{-}=\left(q^{-}, \dot{q}^{-}\right), \operatorname{set}\left(\theta^{-}, \dot{\theta}^{-}\right)=\left(c q^{-}, c \dot{q}^{-}\right)$, where $c=\left[\begin{array}{llllll}0 & -1 & 0 & 0 & 0 & 0\end{array}\right]$.

(2) Compute $\left(q^{+}, \dot{q}^{+}\right)$from the impact equations

$$
\left[\begin{array}{c}
q^{+} \\
\dot{q}^{+}
\end{array}\right]=\left[\begin{array}{c}
\Delta_{q}\left(q^{-}\right) \\
\Delta_{\dot{q}}\left(\dot{q}^{-}\right)
\end{array}\right] \text {, and }\left(\theta^{+}, \dot{\theta}^{+}\right)=\left(c q^{+}, c \dot{q}^{+}\right) .
$$

(3) Set up the Bezier polynomials $h_{d}(\theta)$,

$$
\begin{aligned}
& \alpha_{0}=q^{+}, \alpha_{1}=q^{+}+\frac{\theta^{-}-\theta^{+}}{M} \frac{\dot{q}^{+}}{\dot{\theta}^{+}}, \\
& \alpha_{M-1}=q^{-}-\frac{\theta^{-}-\theta^{+}}{M} \frac{\dot{q}^{-}}{\dot{\theta}^{-}} \text {and } \alpha_{M}=q^{-} .
\end{aligned}
$$

(4) Apply MATLAB's ODE45 function to solve the two degree of freedom zero dynamics equations given in Eq. (11), and obtain the walking period $T$, step size $L$, and the resulting state configuration $X_{k+1}^{-}=\left(\hat{q}^{-}, \dot{\hat{q}}^{-}\right)$at the end of the step (i.e., at the moment the biped's swing foot lands on the ground).

(5) Evaluate the cost J, the constraints (i) - (iv), and for (v), test if $\hat{q}^{-}=H q^{-}$and $\dot{\hat{q}}^{-}=H \dot{q}^{-}$, where $H=\operatorname{diag}\{-1,1,1,-1,-1,1\} \quad$. Such a solution

$X^{*}=\left(q^{*-}, \dot{q}^{*-}\right)$ is called a fixed-point.

A fixed-point solution $X^{*}=\left(q^{*-}, \dot{q}^{*-}\right)$ minimizing $J$ is a desired periodic walking cycle (or nominal orbit).

\section{Remark 1:}

The stability of a fixed-point $X^{*}$ can be tested numerically using a linearized Poincare map. The Poincare map $P: S \rightarrow S$, gives a discrete-time control system $\quad x_{k+1}=P\left(x_{k}\right) \quad, \quad$ where as before $S=\left\{(q, \dot{q}) \mid z_{2}(q)=0, x_{2}(q)>0\right\}$ is the switching surface and $x=\left[\begin{array}{llllllll}q_{1} & q_{2} & \cdots & q_{6} & \dot{q}_{1} & \dot{q}_{2} & \cdots & \dot{q}_{6}\end{array}\right]^{T}$ are the state variables. Because the switching surface $S$ is a hyper surface in $R^{12}$, the Poincare map has 11 independent components. Define a projection operator

$$
\Pi(x)=\left[\begin{array}{llllllll}
q_{1} & q_{3} & \cdots & q_{6} & \dot{q}_{1} & \dot{q}_{2} & \cdots & \dot{q}_{6}
\end{array}\right]^{T}
$$

(i.e, $q_{2}$ is eliminated) and define $\delta x_{k}=\Pi\left(x_{k}-x^{*}\right)$. The Poincare map linearized about a fixed-point $x^{*}=\left(q^{*-}, \dot{q}^{*-}\right)$ gives rise to a linearized system, $\delta x_{k+1}=A \delta x_{k}$, where the $11 \times 11$ square matrix $A$ is the Jacobian of the Poincare map and is computed as follows:

$$
A=\left[\begin{array}{llll}
A_{1} & A_{3} & \cdots & A_{12}
\end{array}\right]_{1 \times 11},
$$

where

$$
A_{i}=\Pi\left(\frac{P\left(x^{*}+\Delta x_{i}\right)-P\left(x^{*}-\Delta x_{i}\right)}{2 \Delta x_{i}}\right), i=1,3, \cdots, 12,
$$

and $x^{*} \pm \Delta x_{i} \in S$. We can solve for $\Delta q_{2}$ and set $\Delta x_{i}=\left\{\begin{array}{cc}\Delta q_{i}+\Delta q_{2} & i=1,3, \cdots, 6 \\ \Delta \dot{q}_{i} & i=7, \cdots, 12\end{array}\right.$ in order to satisfy $z_{2}(q)=0$. A fixed-point of the Poincare map is locally exponentially stable, if, and only if, the eigenvalues of A have magnitude strictly less than one.

\section{Remark 2:}

If the eigenvalues of $\mathrm{A}$ do not have magnitude strictly less than one or if the corresponding rate of convergence is not sufficiently rapid, event-based control may be designed and integrated with the continuous, stance phase controller [13]. Indeed, let $\beta$ be a vector of parameters that are held constant during the stance phase and updated at each impact. The parameters could be a subset of the parameters used to specify the virtual constraints, $\alpha_{0}, \cdots, \alpha_{M}$, or an auxiliary set of parameters introduced by defining, for example

$$
y_{4 \times 1}=h(q, \beta)=q_{a}-\left(h_{d}(\theta)+\beta\right),
$$

or a combination of these. The Poincare map can then be viewed as a nonlinear control system on $\mathrm{S}$ with inputs $\beta$, namely $x_{k+1}=P\left(x_{k}, \beta_{k}\right)$. Linearizing this nonlinear system about the fixed point $x^{*}=\left(q^{*^{-}}, \dot{q}^{*^{-}}\right)$and the nominal parameter value $\beta^{*}$ leads to

$$
\delta x_{k+1}=A \delta x_{k}+B \delta \beta_{k},
$$

where $\delta \beta=\beta-\beta^{*}$ and $B$ is the Jacobian of $\mathrm{P}$ with respect to $\beta$. Designing a feedback matrix $\delta \beta=F \delta x$ such that the eigenvalues of $(A+B F)$ have magnitude strictly less than one will exponentially stabilize the fixed point $x^{*}$.

\section{Simulation Results}

The physical parameters of the $3 \mathrm{D}$ biped were chosen as in Table 1. The parameters result in the center of gravity of the biped being located near the midpoint of the hips.

Table 1. Parameters for the 3D bipedal robot of Fig. 1 (in MKS)

\begin{tabular}{|c|c|c|c|c|c|c|}
\hline $\mathrm{g}$ & $\mathrm{W}$ & $\mathrm{L} 1$ & $\mathrm{~L} 2$ & $\mathrm{~m} 1$ & $\mathrm{~m} 2$ & $\mathrm{M}$ \\
\hline 9.81 & 0.15 & 0.55 & 0.05 & 1.75 & 5.5 & 9.0 \\
\hline
\end{tabular}

For these parameters, a periodic orbit was computed following Section 4 using degree 3 Bezier polynomials in the 
virtual constraints. We obtained a periodic and symmetric fixed-point solution $X^{*}=\left(q^{*-}, \dot{q}^{*-}\right)$, where

$$
\begin{aligned}
q^{*-} & =\left[\begin{array}{lllllll}
0.0 & -0.10842 & -0.66840 & 0.0 & 0.0 & -0.88523
\end{array}\right]^{T} \\
\dot{q}^{*-} & =\left[\begin{array}{llllll}
0.13779 & -1.9120 & 0.30038 & 0.0 & 0.0 & 2.0085
\end{array}\right]^{T} ;
\end{aligned}
$$

the corresponding Bezier coefficients are

$$
\alpha^{*}=\left[\begin{array}{cccc}
-0.88523 & -1.3439 & -0.67975 & -0.66840 \\
0.0 & 0.0035691 & 0.0 & 0.0 \\
0.0 & 0.021127 & 0.0 & 0.0 \\
-0.66840 & -1.15960 & -0.96116 & -0.88523
\end{array}\right] .
$$

The walking cycle has a period of $T=0.1335$ seconds, a step size of $L=0.1168 \mathrm{~m}$, and an average walking speed of $0.875 \mathrm{~m} / \mathrm{sec}$. The nominal gait's joint profiles and angular velocities over two complete steps are shown in Figs. 3 and 4, respectively. Fig. 5 shows the profile of the reaction force $F_{1}$ on the stance foot over two nominal walking cycles.
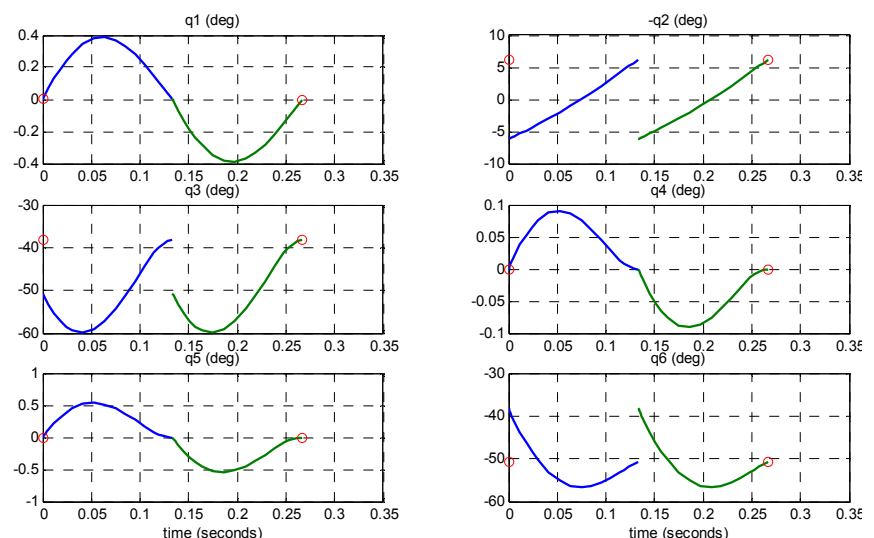

Figure 3. Joint profiles of the obtained fixed-point $X^{*}$ over two walking cycles, where the small circles represent $q^{*}$.
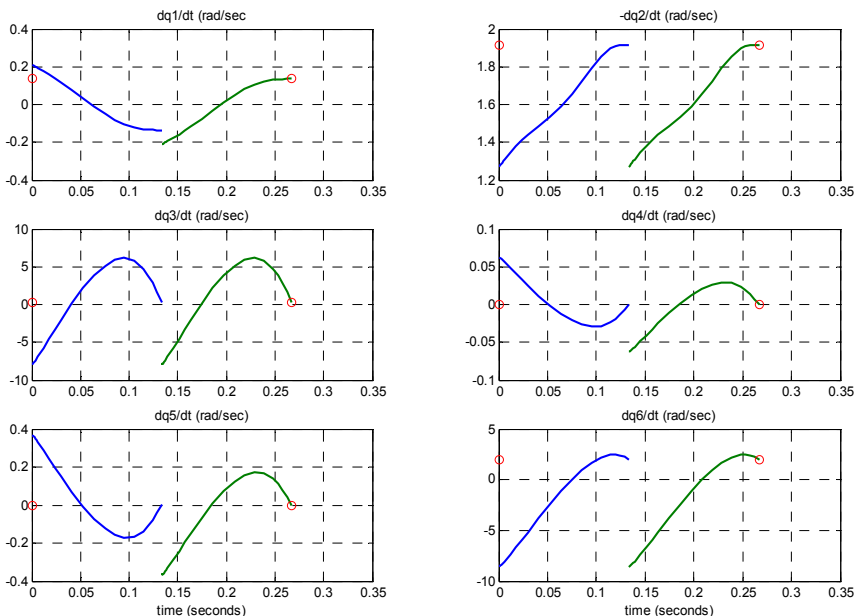

Figure 4. Joint rate profiles of the found fixed-point $X^{*}$ over two walking cycles, where the small circles represent $\dot{q}^{*-}$.
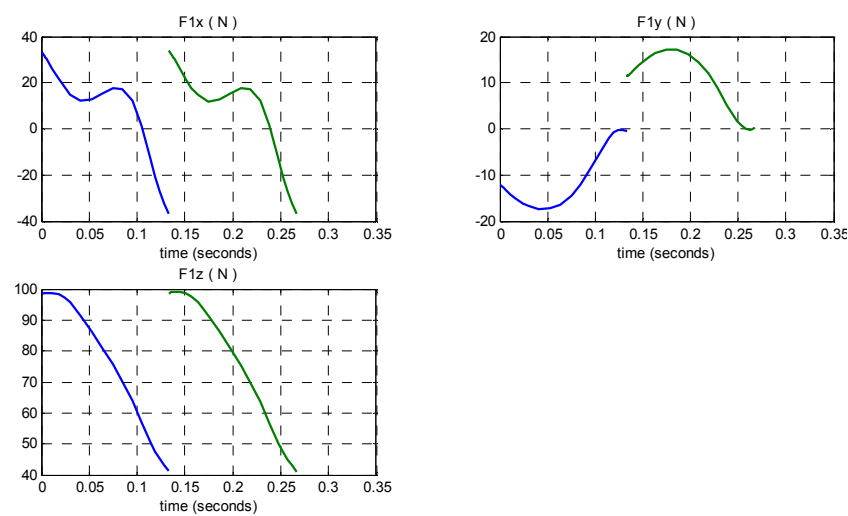

Figure 5. The reaction force $F_{1}$ on the stance foot over two nominal walking cycles.

Having obtained the virtual constraints, $y=q_{a}-h_{d}(\theta)$, a control law for the full model of the 3D biped was obtained as in Section 3, with a feedforward computed torque component and PD correction terms,

$$
u=u^{*}-\left(\frac{\partial h}{\partial q} D^{-1} B\right)^{-1}\left(\frac{K_{p}}{\varepsilon^{2}} y+\frac{K_{d}}{\varepsilon} \dot{y}\right) .
$$

The PD control gains used are listed in Table 2. Fig. 6 shows a snapshot taken at $t=9.887$ seconds from an animation of the closed-loop system initialized at the fixed point $X^{*}$ at time $t=0$. The computed eigenvalues of the linearized Poincare map are listed in Table 3. All the eigenvalues have magnitude less than 1.0, the obtained nominal orbit $X^{*}$ is locally exponentially stable. To demonstrate the orbit's local stability, Fig. 7 shows the output function and its derivative for the 3D biped's full-model in closed-loop with an initial state perturbed from the fixed-point $X^{*}$. To demonstrate the eventbased control, Fig. 8 shows the output function and its derivative (starting from the same initial state as Fig. 7) when an event-based DLQR controller is used, where $\beta$ is chosen as the constant offsets of the actuated angles $q_{a}$. Note the output $y$ is rapidly convergent to zero values in Fig. 8 .

Table 2. PD control gains

\begin{tabular}{|c|c|c|}
\hline$K_{P}$ & $K_{d}$ & $\varepsilon$ \\
\hline 50.0 & 10.0 & 0.1 \\
\hline
\end{tabular}

Table 3. Eigenvalues of the linearized Poincare map

\begin{tabular}{|c|c|c|}
\hline$i$ & $\lambda_{i}$ & $\left|\lambda_{i}\right|$ \\
\hline 1,2 & $-0.2525 \pm 0.9571 \mathrm{i}$ & 0.9899 \\
\hline 3 & 0.8842 & 0.8842 \\
\hline 4 & -0.0029 & 0.0029 \\
\hline 5 & 0.0021 & 0.0021 \\
\hline 6,7 & $-0.0012 \pm 0.0008 \mathrm{i}$ & 0.0015 \\
\hline 8 & -0.0009 & 0.0009 \\
\hline 9 & 0.0006 & 0.0006 \\
\hline 10 & -0.0003 & 0.0003 \\
\hline 11 & 0.0 & 0.0 \\
\hline
\end{tabular}




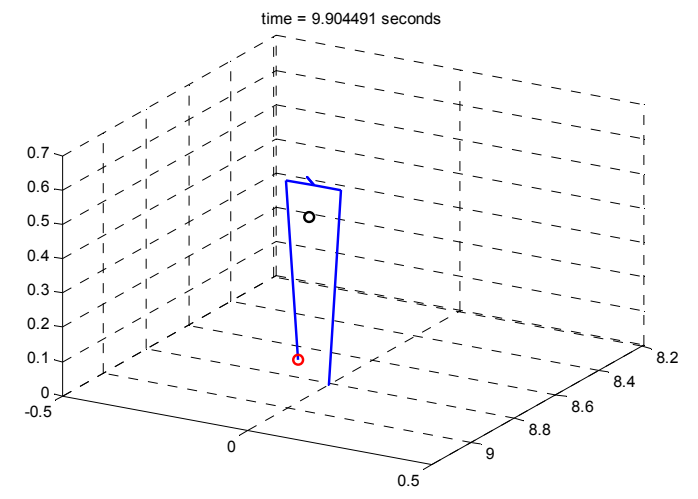

Figure 6. The snapshot of an animation of the 3D biped fullmodel walking control motion taken at the moment $t=9.9045$ seconds.
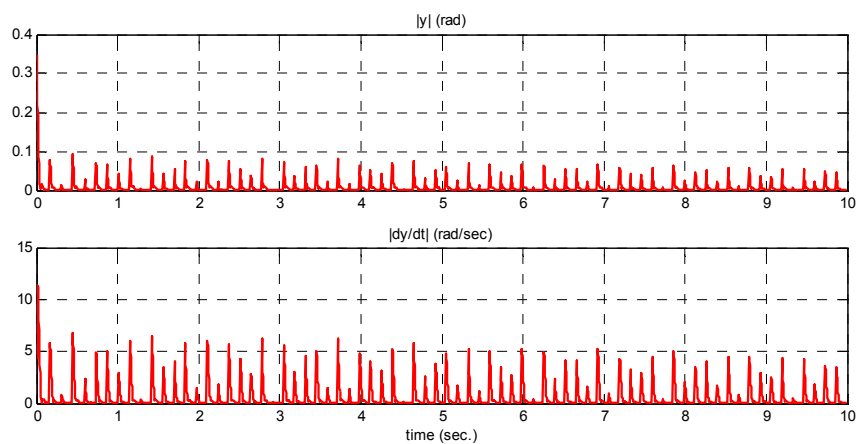

Figure 7. The output function and its derivative for the 3D biped's full-model under closed-loop walking control, with the initial condition perturbed from $X^{*}$.
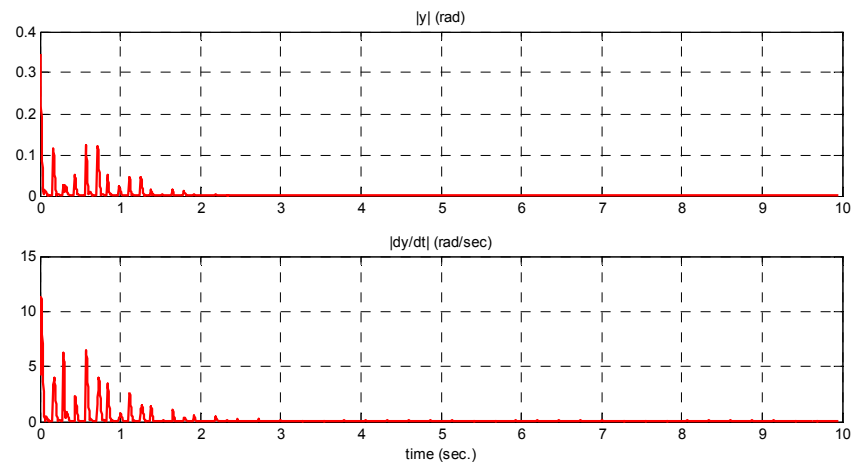

Figure 8. The output function and its derivative, starting from the same initial state as Fig. 7, when an event-based DLQR controller is applied.

\section{CONCLUSIONS}

A simple 3D bipedal model has been studied, with the objective of developing a time-invariant feedback control law that induces asymptotically stable walking, without relying on the use of large feet. For this reason, the biped was assumed to have point feet with no actuation between the feet and ground. Inspired by its success in solving similar problems for planar robots, the method of virtual constraints was applied to the $3 \mathrm{D}$ robot, with the virtual constraints chosen via optimization as suggested in [4]. For a biped consisting of three links connected to form two legs without knees and a torso, and for a nominal set of parameters, the method resulted in a locally exponentially stable walking motion of approximately one and a half body lengths per second. For this robot, no additional event-based control was necessary to stabilize the obtained walking motion, but the authors expect that this will not always be the case.

This case study was completed by following guidelines suggested by a detailed theory of feedback control of planar robots that has been developed over the past eight years. There was no a priori guarantee that a stable walking motion would be obtained. It is important to develop a careful theory for the case of spatial robots so that when the procedure followed here fails, one would know why it fails and, hopefully, how to modify it.

\section{ACKNOWLEDGMENT}

The work of C.L. Shih is supported by Taiwan NSC grant NSC-95-2918-I-011-002. The work of J.W. Grizzle is supported by NSF grant ECS-0600869.

\section{REFERENCES}

[1] J.W. Grizzle, G. Abba, and F. Plestan, "Asymptotically Stable Walking for Biped Robots: Analysis via Systems with Impulse Effects," IEEE Transactions on Automatic Control, Vol. 46, pp. 51-46, January 2001.

[2] F. Plestan, J. W. Grizzle, and E.R. Westervelt, and G. Abba, "Stable Walking of a 7-DOF Biped Robot," IEEE Transactions on Robotics and Automation, Vol. 19, No. 4, pp. 653-668, August 2003.

[3] C. Chevallereau, and etc. "RABBIT: A Testbed for Advanced Control Theory," IEEE Control Systems Magazine, Vol. 23, No. 5, pp. 57-79, October 2003.

[4] E.R. Westervelt, J.W. Grizzle, and D.E. Koditschek, "Hybrid Zero Dynamics of Planar Biped Walkers," IEEE Transaction on Automatic Control, Vol. 48, No. 1, January 2003, pp. 42-56.

[5] T. Fukuda, M. Doi, Hasegawa, and H. Kajima, Fast Motions in Biomechanics and Robotics, chapter Multi-Locomotion Control of Biped Locomotion and Brachiation Robot, pp. 121-145, Springer-Verlag, Heidelberg, Germany, 2006.

[6] Arthur D. Kuo, "Stabilization of Lateral Motion in Passive Dynamic Walking," The international Journal of Robotics Research, Vol. 18, No. 9, pp. 917-930, 1999.

[7] G. Song and M. Zefran, "Underactuated Dynamic Three-Dimensional Bipedal Walking," Proceeding of the 2006 IEEE International Conference on Robotics and Automation, Orlando, Florida, May 2006, pp. 854-859.

[8] S. H. Collins, A. Ruina, R. Tedrake, and M. Wisse, "Efficient bipedal robots based on passive-dynamic walkers," Science, 307:1082-85, 2005.

[9] M.W. Spong and F. Bullo, "Controlled Symmetries and Passive Walking," IEEE Transactions on Automatic Control, Vol. 50, No. 7, pp: 1025-1031, July, 2005.

[10] A. D. Ames and R. D. Gregg, "Stably extending two-dimensional bipedal walking to three," In Proc. of the 2007 American Control Conference, New York, NY, 2007.

[11] M. Vukobratovic, B. Borovac, and V. Potkonjak, "ZMP: A Review of Some Basic Misunderstandings," International Journal of Humanoid Robotics, Vol. 3, No. 2, pp. 153-175, June 2006.

[12] B. Morris and J.W. Grizzle, "A Restricted Poincare Map for Determining Exponentially Stable Periodic Orbits in Systems with Impulse Effects: Application to Bipedal Robots," 2005-CDC, Seville, Spain, December, 2005.

[13] J.W. Grizzle, "Remarks on Event-Based Stabilization of Periodic Orbits in Systems with Impulse Effects," Second International Symposium on Communication, Control and Signal Processing, March 2006, Marrakech, Morocco. 